\title{
DETECTING OPEN CRAKS INSIDE BEAMS USING THE BEM AND WAVELET TRANSFORM
}

\author{
R. S. Y. C. Silva ${ }^{1}$, L. M. Bezerra ${ }^{2}$, L. A. P. Peña ${ }^{1}$ \\ ${ }^{1}$ Department of Civil Engineering, University of Brasilia (ramon@unb.br)
}

\begin{abstract}
.
There are several techniques of non-destructive damage detection in structures. However, these techniques are expensive and require an accurate examination of large extension of the structure under analysis. The numerical techniques can be helpful for non-destructive examination of structures. Such techniques may show a possible location of damage and thus decrease substantially the area to be examined and, consequently, may turn the non-destructive tests less expensive. Among many numerical methods, the boundary element method presents positive attributes that can be used in formulations proposed to solve the problem of detecting damages in structures such as beams. Generally, the problem of detecting damages is a ill-posed problem and the common approach used is the comparison of signatures obtained before and after the onset of damage. The signatures maybe defined in terms of displacements, mode shapes, strains or stresses. In this article, the methods based on Wavelet Transform associated with the boundary element technique will be combined with the scope to detect the position of a damage just using the damaged response of the actual structure. After presenting the formulation, numerical applications for detecting damages in beams under static loads are presented and discussed.
\end{abstract}

Keywords: damage, beam, wavelet.

\section{INTRODUCTION}

Most methods for damage detection are based on the comparison of signatures written in terms of displacements, strains, stresses, natural frequencies, mode shapes, damping ratio, and so on, obtained before and after the coming out of a damage. These methods are based on the assumption that a structural damage may cause significant variation in structural parameters such as mass, stiffness, or flexibility and those variations may cause substantial changes in structural responses, such as natural frequencies, mode shapes, damping ratio. The variations of such responses may be expressed by a signature which is just a residual function that compares the results obtained in a conceived mathematical model with the data (displacements, strains, stresses, natural frequencies, mode shapes, damping ratio, etc...) measured in the real structure.

The formulations based in such signatures search the minimum of the residual function with any algorithm, that may be deterministic, such as the conjugate gradient method, the BFGS method, Marquad's methods [1,2], or non-determinist algorithms such as the generic algorithm, ant's colony algorithm [3], and so on.

When such residual function is at a minimum, one can conclude that the mathematical 
model is well expressing the damage. Such methods gained popularities due to significant advances in the experimental methods and technologies for monitoring structures [4,5]. However, such approaches need a comparison between two states, which means more work and the storage of the data between the two states. Methods which can detect damage with only information obtained from the damage condition of the structure are more advantageous since their condition before damage is rarely known. In this context, the application of the wavelet based methods can be useful. These methods detect the singularities present in the static and modal response caused by damage and therefore they do not require the condition of the structure before damage. It is also necessary to determine a numerical procedure for the simulation of cracked structures. In this way, evaluation of the damage detection methods can be practical [4].

This paper presents two numerical examples using boundary elements and Wavelet Transform for damage detection in a cantilever beam. The modeling of damage is done in boundary elements by means of program Elast_qua [8] developed in MATLAB. The static response of the structure with simulated damage is used in the analysis to detect the location of damage using the Wavelet Transform.

\section{THE BOUNDARY ELEMENT FORMULATION}

The Boundary Element Method (BEM) possesses many encouraging attributes such as: (a) Reduction by one of the problem dimensionality; 2D problems are reduced to 1D. (b) Only the boundary of the structure may possibly be discretized. (c) The domain of the continuum does not have to be divided into elements and nodes. (d) Structural response at all the internal nodes, as in FEM, can be waived. (e) Discretization limited only to the structure boundaries leads to a system of equation with smaller number of degrees of freedom. Those features, among others, are advantageous for ill-posed problems [6], for example, the numerical stability in the solution of ill-posed problems using BEM is much better than via FEM. The BEM can be introduced, among other approach, with the weighted residual technique or with the reciprocal principle theorem, as:

$$
\int_{\Omega} \sigma_{\mathrm{ij}} \varepsilon_{\mathrm{ij}}^{*} \mathrm{~d} \Omega=\int_{\Omega} \sigma_{i j}^{*} \varepsilon_{\mathrm{ij}} \mathrm{d} \Omega
$$

Where $\Omega$ is the structure mathematical domain with boundary, see Fig.1, $\sigma_{\mathrm{ij}}, \sigma_{\mathrm{ij}}^{*}, \varepsilon_{\mathrm{ij}}$ and $\varepsilon_{\mathrm{ij}}^{*}$ are, respectively, stresses and strains in the same body $\Omega$ in equilibrium state under different loads assigned without and with the starred symbol $(*)$. After integrating by parts Equation (1), and taking into consideration the equilibrium equation in the continuum, written $a s \sigma_{\mathrm{ij}, \mathrm{j}}^{*}+\mathrm{b}_{\mathrm{j}}^{*}=0$ (for $\mathrm{b}_{\mathrm{j}}^{*}$ as a unit point load - Dirac delta function), the Somigliana's Identity [7] can be easily obtained as:

$$
\int_{\Omega} b_{k}^{*} u_{k} d \Omega+\oint_{\Gamma} t_{k}^{*} u_{k} d \Gamma=\int_{\Omega} b_{k} u_{k}^{*} d \Omega+\oint_{\Gamma} t_{k} u_{k}^{*} d \Gamma
$$


In Eq. (2), $\mathrm{t}_{\mathrm{k}}, \mathrm{u}_{\mathrm{k}}$ and $\Gamma$ are, respectively, loads, displacements, and boundary of the body. Using the Dirac delta function mathematical properties, Eq. (2) can be rewritten as:

$$
\begin{gathered}
u_{i}=\oint_{\Gamma} u_{i j}^{*} t_{j} d \Gamma-\oint_{\Gamma} t_{i j}^{*} u_{j} d \Gamma \\
\operatorname{With}\left\{\begin{array}{l}
\mathrm{u}_{\mathrm{ij}}^{*}=-\frac{1}{8 \pi \mu(1-v)}\left[(3-4 v) \delta_{\mathrm{ij}} \log \mathrm{R}-\frac{\mathrm{Y}_{\mathrm{i}} \mathrm{Y}_{\mathrm{k}}}{\mathrm{R}^{2}}\right] \\
\mathrm{t}_{\mathrm{ij}}^{*}=-\frac{1}{4 \pi \mathrm{R}^{2}(1-v)}\left\{(1-2 v)\left(\mathrm{n}_{\mathrm{k}} \mathrm{Y}_{\mathrm{i}}-\mathrm{n}_{\mathrm{i}} \mathrm{Y}_{\mathrm{k}}\right)+\left[(1-2 v) \delta_{\mathrm{ik}}+\frac{2 \mathrm{Y}_{\mathrm{i}} \mathrm{Y}_{\mathrm{k}}}{\mathrm{R}^{2}}\right] \mathrm{Y}_{\mathrm{j}} \mathrm{n}_{\mathrm{j}}\right\}
\end{array}\right.
\end{gathered}
$$

Equation (4) represents the fundamental solution [7] where $v, \mu$ and $E$, are, respectively; the Poisson's ratio, the shear modulus, and the modulus of Elasticity. The term $R^{2}=Y_{i} Y_{i}$. Moreover, $Y_{i}=x_{i}-\xi_{i}$ represents the distance between the field point $x_{i}$ and the load point $\xi_{i}$. The term $n_{i}$ is the outward normal at boundary $\Gamma$ - for more details see [7]. The close solution for obtaining the displacement $u_{i}$ in the integral Equation (3) of Somigliana's Identity is a very difficult task and only attainable for simple geometries and boundary conditions. The BEM provides a numerical approach for the solution of Eq. (3) in the following steps: (a) The boundary $\Gamma$ is discretized into a series of $\mathrm{N}$ boundary elements $\Gamma=\sum_{i=1}^{N} \Gamma_{i}$ defined over a set of nodes. (b) Eq. (3) is applied in the discretized form to each nodal point of the boundary and the integrals are computed by numerical quadrature scheme over each boundary element. (c) A system of $M$ linear algebraic equations involving the set of $M_{1}$ nodal traction and $\mathrm{M}_{2}$ nodal displacements is obtained. (d) The boundary conditions are imposed and consequently the $\mathrm{M}$ modal values (tractions or displacements in each direction per node) are prescribed. (e) The system of $M$ equations is solved by standard methods to obtain the remaining boundary data, for more details see [7].

In this work the elements used are for quadratic boundary elements implemented in the MatLab program [8]. In the program, the displacement $u_{i}$ in the integral Equation (3) is obtained for the boundary nodes. After that, any internal point can have its displacements calculated [7]. Such displacements are the quantities that will be considered in this work and will be wavelet transformed.

\section{WAVELETS THEORY}

Considering a signal of interest in the time or space domain and $\psi(\mathrm{t})$ the values of wavelet function in the time and frequency domains. The wavelets are generated from the mother wavelet $\psi(\mathrm{t})$ by translation and dilation, as follow below:

$$
\psi_{a, b}(t)=\frac{1}{\sqrt{a}} \psi\left(\frac{t-b}{a}\right)
$$


Where $\mathrm{a}$ and $\mathrm{b}$ are integer numbers which represents the dilation and translation parameters respectively. The wavelet transform of a signal $\mathrm{f}(\mathrm{t})$ is defined by:

$$
C_{a, b}=C_{a, b}\left(t_{o}\right)=\int_{-\infty}^{\infty} f(t) \Psi_{a, b}\left(t_{o}\right) d t
$$

The results of this transformation are called wavelet coefficients and show how well the function correlates with the signal. These wavelet coefficients are very sensitive to discontinuities and singularities present in the analyzed signal. Considering this property, it was found that damage due to a sudden loss of stiffness can be detected through mode shapes with wavelet coefficients which achieve large amplitudes like a spike or an impulse in the damage location. This perturbation of wavelet coefficients due to this damage is clearer in the finest scales of the wavelet transform. This procedure is the basis of the wavelet transform damage detection [5].

\section{NUMERICAL EXAMPLES}

This section presents the boundary element model of cantilever beams modeled using quadratic elements of the Elast_qua[8] program. This element has three nodes and three degrees of freedom by node: translations in $\mathrm{x}, \mathrm{y}$ and traction.

The static responses (displacements) were analyzed in MATLAB program to compute the wavelet coefficients using the Biorthogonal 3.7 mother-wavelet.

\subsection{Cantilever beam - case 1}

In case 1 , the cantilever beam used in the analysis was submitted to a load $F=500 \mathrm{kN}$ in the free end and to a transverse crack a' $=0,025 \mathrm{~m}$ positioned at $d=0,25 \mathrm{~m}(\mathrm{~L} / 2)$ from the left end, see Fig. 1. The material and geometric properties of the beams analyzed are shown in Table1.

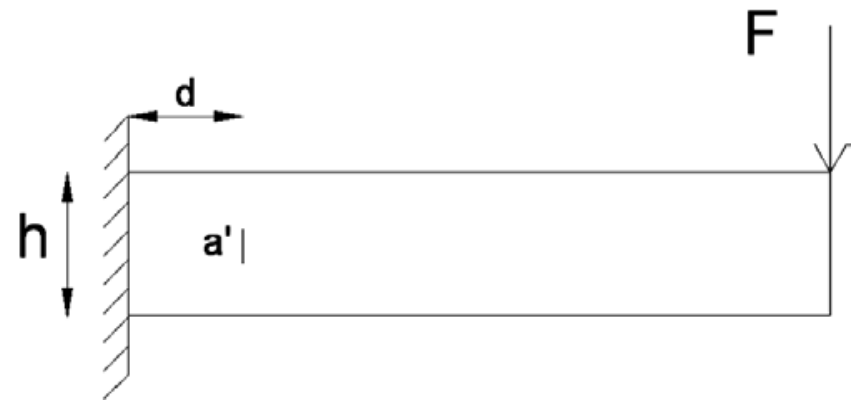

Figure 1. Cantilever beam analyzed case 1. 
Table 1: Geometric and materials properties of beam.

\begin{tabular}{lccc}
\hline Properties & Symbol & Value & Unity \\
\hline Beam width & $\mathrm{B}$ & 0.10 & $\mathrm{~m}$ \\
Beam height & $\mathrm{h}$ & 0.10 & $\mathrm{~m}$ \\
Area & $\mathrm{S}$ & 0.01 & $\mathrm{~m}^{2}$ \\
Beam length & $\mathrm{L}$ & 0.50 & $\mathrm{~m}$ \\
Modulus of elasticity & $\mathrm{E}$ & 200.00 & $\mathrm{GPa}$ \\
Poisson's coefficient & $\mathrm{v}$ & 0.30 & - \\
\hline
\end{tabular}

The boundary element model of cantilever beam was discretized in 25 elements and 204 nodes. The damage was simulated defining the crack in the clockwise direction and as a hole that is an open crack, furthermore, the boundary conditions were applied in the all nodes of left end restricting the degrees of freedom in $\mathrm{x}$ and $\mathrm{y}$ direction, see Fig. 2.

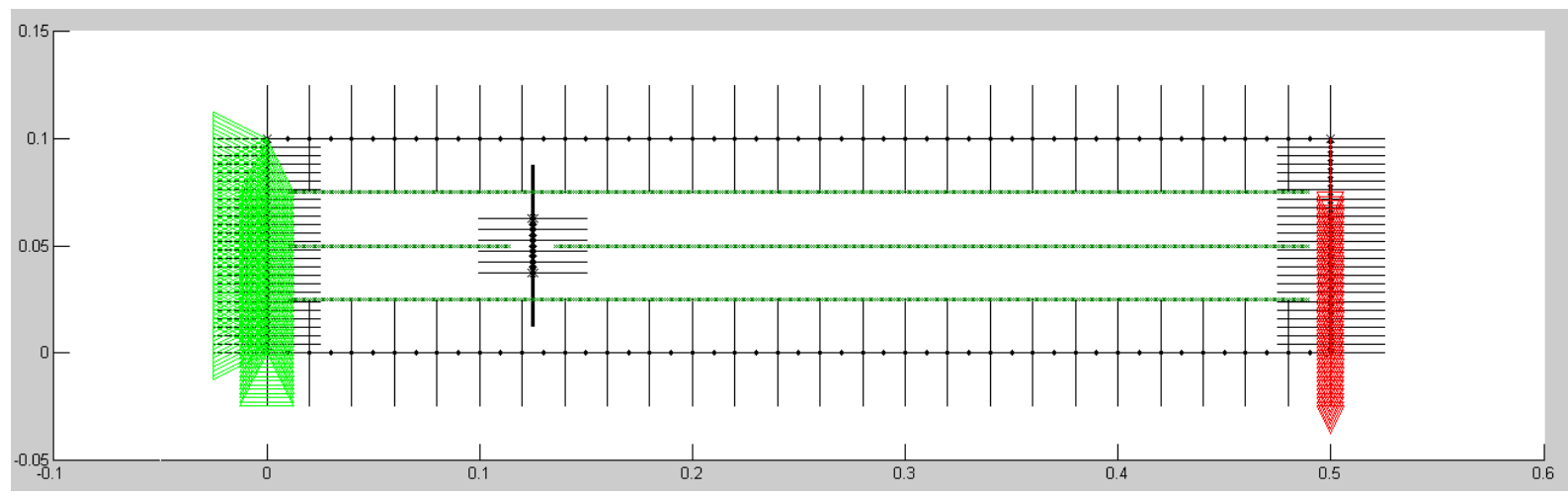

Figure 2. Boundary element model of cantilever beam case 1.

Four hundred and eighty internal points were created at 1/3 of beam height to be used to plot the deflection of beam and to applicate the wavelet transform in the static response (nodal displacements).The deformed shape and the deflection of beam with crack and without crack obtained in the program are presented in the Fig. 3 and Fig. 4 respectively.

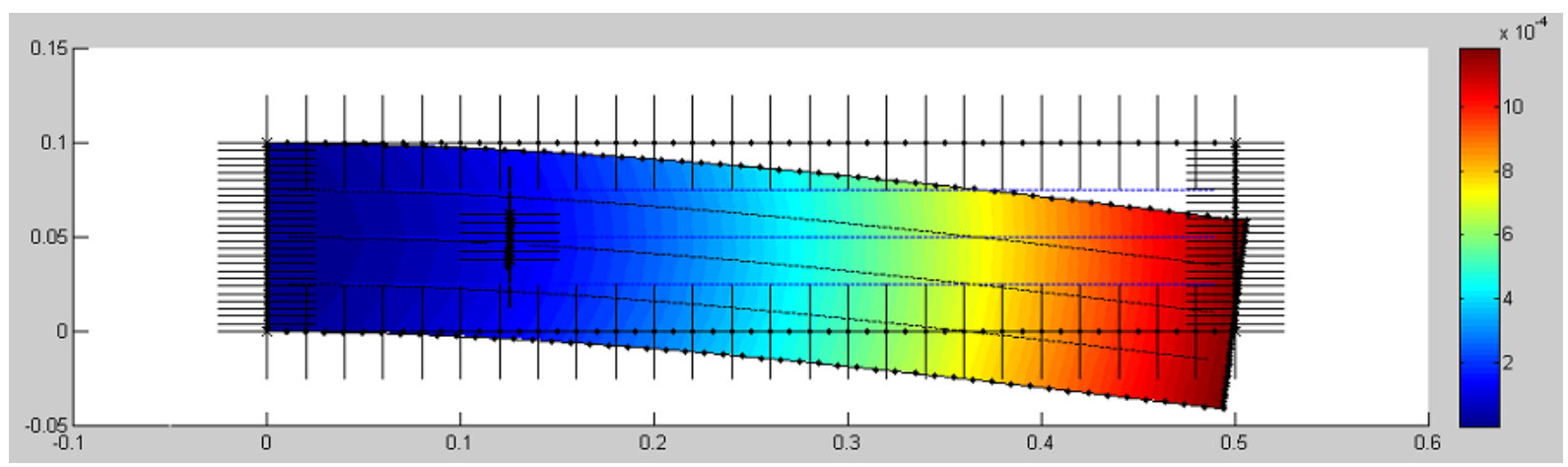

Figure 3. Deformed shape with crack case 1 


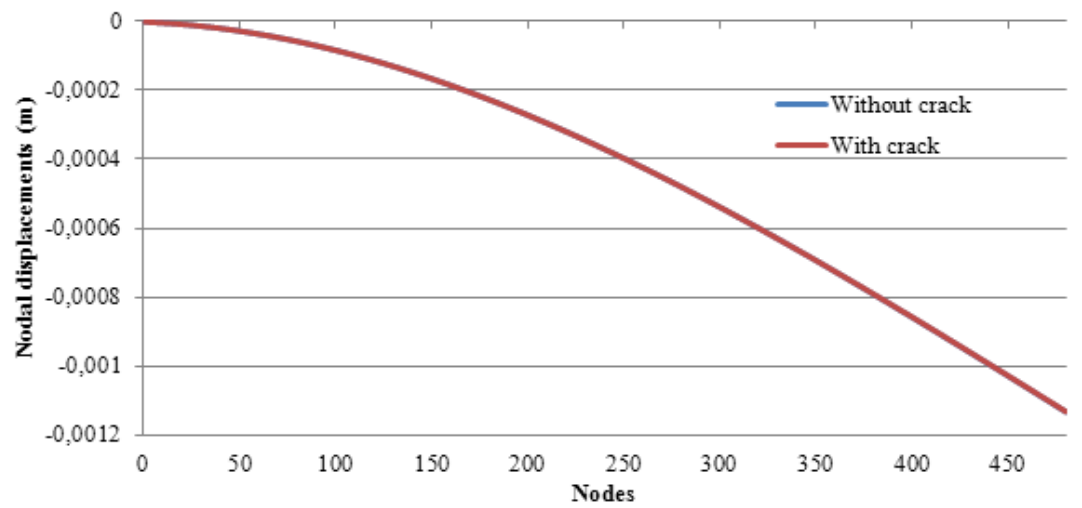

Figure 4. Deflection of cantilever beam case 1.

The variations in the nodal displacements in the cantilever beam with and without crack could not be distinguished satisfactorily to provide the case with the damage; it may be because the damage is too small.

The wavelet transform was applied in the signal of displacements obtained in the internal points using the wavetoolbox of MATLAB to compute the wavelet coefficients for motherwavelet Biorthogonal 3.7(bior3.7). The Fig. 5 shows the result of this wavelet transform application.

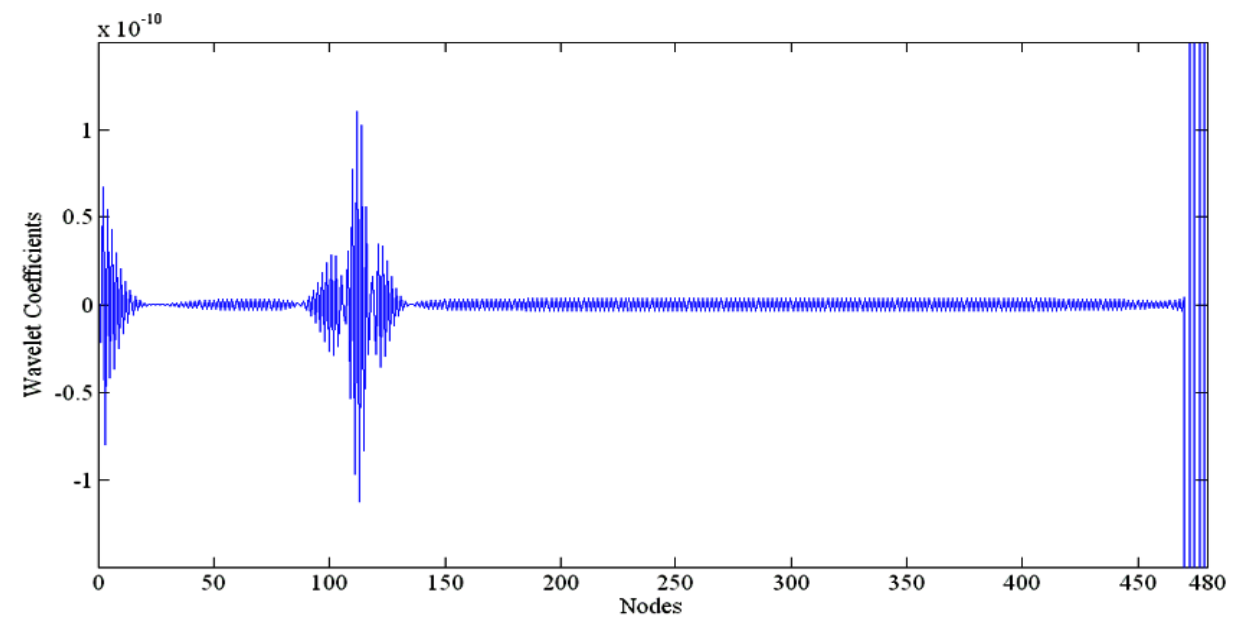

Figure 5. Wavelet coefficients using bior3.7 case 1.

The bior3.7 mother-wavelet were able to detect the exact position of damage, moreover, the graphics presented perturbations in the ends due to geometric discontinuities. These perturbations were also observed in others papers that used finite element method to detect damages in beams $[9,10]$. 


\subsection{Cantilever beam - case 2}

In case 2, the crack was positioned $d=0,375 \mathrm{~m}$ from the left end, see Fig. 6 . The material and geometric properties of the beam analyzed are the same of case 1:

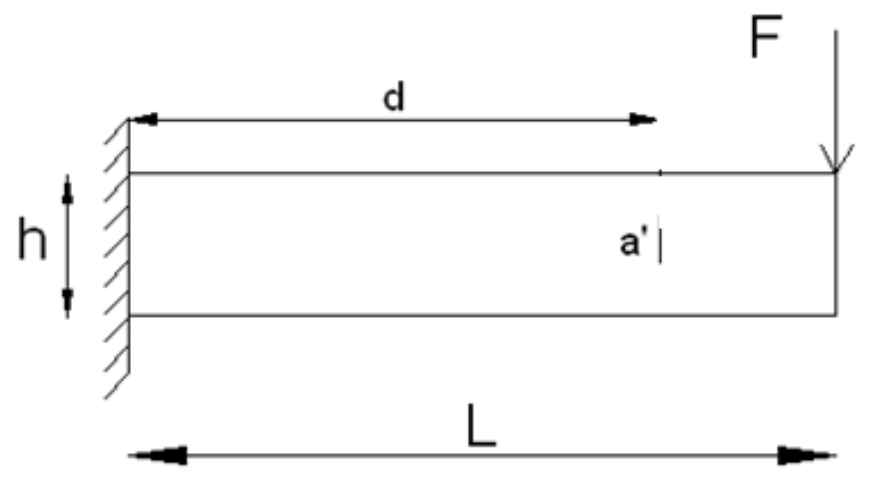

Figure 6. Cantilever beam analyzed case 2.

The boundary element model of cantilever beam was discretized in 25 elements and 204 nodes. The beam with the boundary conditions is presented in Fig. 7.

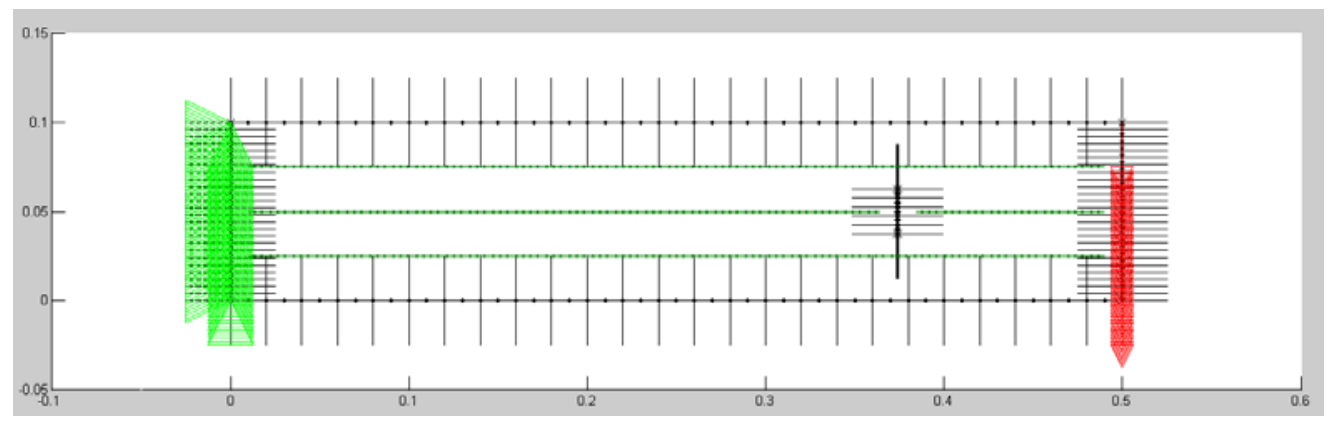

Figure 7. Boundary element model of cantilever beam case 2

Four hundred and eighty internal points were created at 1/3 of beam height to be used to plot the deflection of beam and to applicate the wavelet transform in the static response (nodal displacements).The deformed and the deflection of the beam in case 2 presented similar results to case 1. The results are presented in the Fig. 8 and Fig. 9.

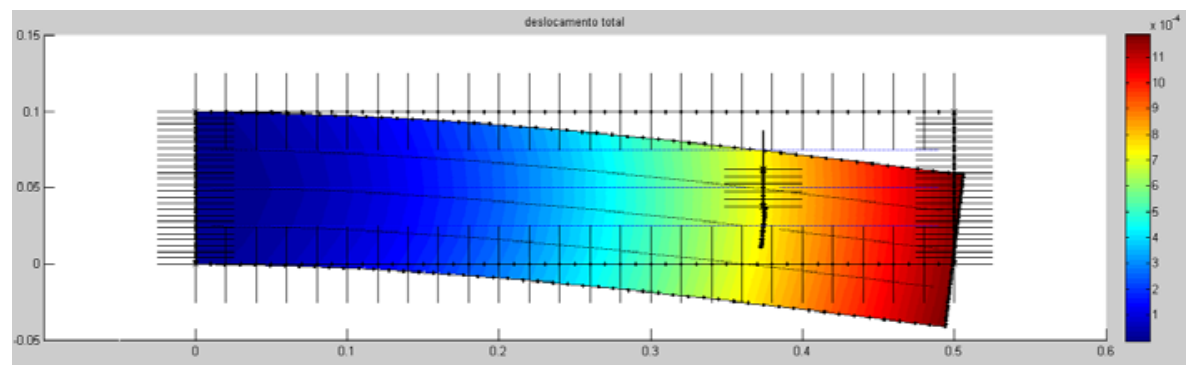

Figure 8. Deformed shape case 2. 


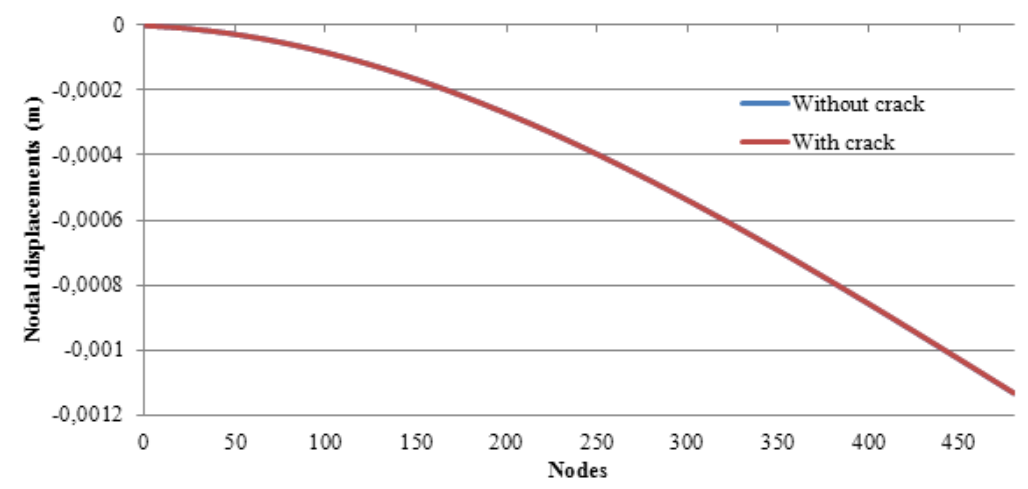

Figure 9. Deflection of cantilever beam case 2.

Again, the variations in the nodal displacements in the cantilever beam with and without crack could not be distinguished satisfactorily; it may be because the damage is too small.

The wavelet transform was applied in the signal of displacements obtained in the internal points using the wavetoolbox of MATLAB to compute the wavelet coefficients for motherwavelet Biorthogonal 3.7(bior3.7). The Fig. 10 shows the result.

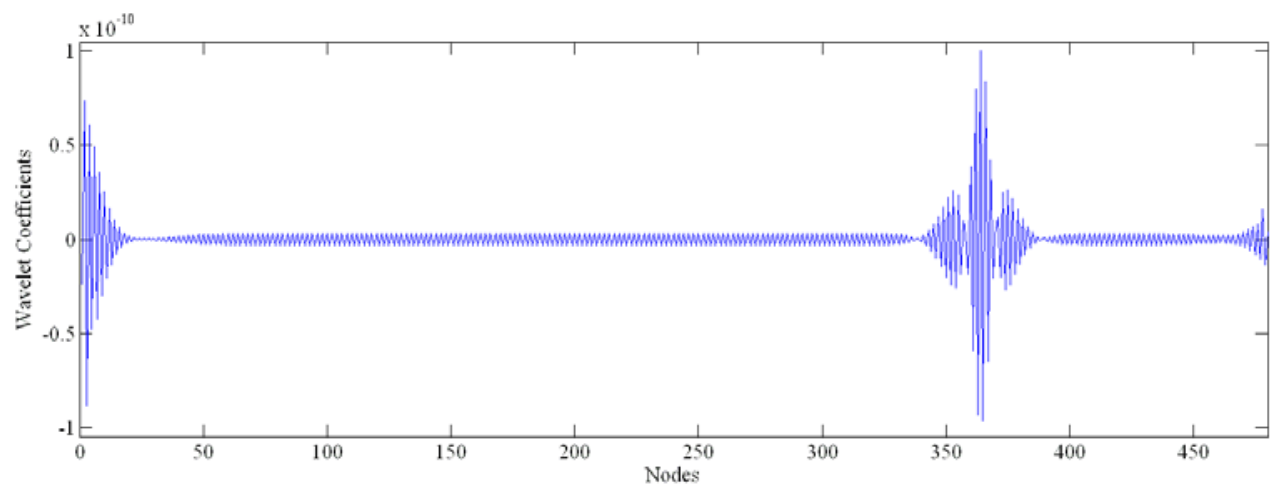

Figure 10. Wavelet coefficients using bior3.7 case 2

\section{CONCLUSIONS}

This paper presented a new methodology applied to the inverse problem of damage detection using boundary elements and wavelet transform using a cantilever beam with a crack at two different positions.

The wavelet transform applied in the static signals could detect the exact position of damages and also, singularities due to geometric discontinuities.

The use of boundary element method could be an alternative in the getting of numerical response for damaged structures to be used in the damage detection process. 


\section{REFERENCES}

[1] P. E. Gill, W. Murray, M. H. Write, "Pratical Optimization”. London, Academic Press(1982).

[2] R. L. Fox, “Optimization Method for Engineering Design”. Massachusetts: AddisonWesley(1971).

[3] M. Dorigo, T. Stutzle, “Ant Colony Optimization”. Wiley-ISTE.(1971).

[4] E S.Estrada, "Damage detection methods in bridges trough vibration monitoring: evaluation and application”. PhD. Thesis, Universityof Minho, 2008.

[5] R. S. Y. C. Silva, "Determinação de patologias estruturais utilizando modelagem numérica e transformadas de wavelet”. MS. Thesis. University of Brasilia. Department of Civil Engineering, 2011.

[6] B. Dorri, “Solution of Inverse Heat Conduction Problems Using Boundary Integral Method”. General Eletric. CRD Report, 1987.

[7] C. A.Brebbia,J. Dominguez, "Boundary Elements An Introductory Course”. WIT Press/Computational Mechanics,1992.

[8] F. A. G. Lourenço. “Análise de Reparos de Trincas Através do Método dos Elementos de Contorno” PhD. Thesis. University of Campinas, 2000. 